RADIO SCIENCE Journal of Research NBS/USNC-URSI

Vol. 69D, No. 5, May 1965

\title{
Preface to the Fourth Group of "Waves in Plasma" Papers
}

This issue of Radio Science contains a fourth group of papers dealing with the general subject of "Waves in Plasma." The first six papers deal with plasmas which are effectively at zero temperature (i.e., cold), while the latter four papers are concerned with various phenomena in warm plasma.

Radiation from a magnetic dipole in magnetoplasma media is considered by Motz, who extends his earlier work on the subject. A related topic is discussed by Mittra and Duff who demonstrate the important role played by the dispersion surface in characterizing the radiation pattern.

The following three papers (Burman and Gould, Burman, and Galejs) deal with various aspects of reflection from inhomogeneous plasma. The paper by Galejs is a thorough analytical treatment of ELF and VLF propagation in the earth ionosphere waveguide when the earth's magnetic field may be regarded as vertical.

The experimental determination of the electron density profile in a cylindrical plasma column is discussed in an interesting paper by Aničin. The method makes use of the refractive or bending characteristics of the plasma for an incident microwave beam.

Waves supported by a uniform plasma slab is considered by Caron, who includes the effect of electroacoustic type waves. He shows that certain characteristics of the surface and leaky waves are critically dependent on the finite electron temperature.

Transmission and reflection from a plasma slab are treated by Taylor using the relativistic form of the Vlasov equation. It is shown that the temperature dependence is a relativistic effect. The author also emphasizes that care should be taken in going to the zero temperature limit because of the nonuniform character of the transition.

Radiation from gyrating electrons in a magnetoplasma is treated exhaustively by Liemohn. In particular, he shows that most of the energy is radiated along wave normals at large angles to the magnetic field at frequencies other than the Doppler-shifted fundamental cyclotron harmonic. This is contrary to assumptions of certain of the earlier VLF emission theories.

In the final paper, the effect of heavy ions on the radiation from a moving charge is considered by Seshadri and Tuan. Among other things, it is shown that there is a lower hybrid resonance frequency below which the ordinary mode is not excited.

The next issue of Radio Science will contain the fifth and final grouping of "Waves in Plasma" papers. 